\title{
Winners of national research awards celebrate
}

A celebratory luncheon for the 2018 recipients of the Robin Davies DCP (dental care professionals) Awards took place at Chandos House, London, recently, attended by leaders in dentistry.

The awards are given in partnership between the Oral and Dental Research Trust (ODRT) and Colgate.

They are awarded for research conducted by dental care professionals to support investigations of clinical relevance, particularly in the field of preventive care. The name of the awards changed in 2017 to reflect the contribution of Professor Robin Davies, a vanguard of dental research who inspired and supported many young researchers from all parts of the dental profession.

Drs Lone Lenes and Emanuele Cotroneo from Colgate Scientific Affairs welcomed everyone acknowledging Colgate's long term commitment and pleasure in partnering with the ODRT for the last 15 years.

Sara Hurley, Chief Dental Officer for England then reinforced the importance of a dental team approach and the increasing contribution of DCPs in improving oral health. She also mentioned previous notable awardees whose work has opened up new opportunities within dental education, leadership and community care. Professor Angus Walls, ODRT Chair, introduced the attending 2018 awardees as:

- Lucy Rutter, dual qualified dental hygienist and dental therapist for investigating a mixed methods evaluation of the Leeds
City Council's oral health promotion intervention, 'Choose the Cup Campaign'

- Kimberley Pickering, dental nurse for investigating the attitudes and perceptions of Muay Thai participants on using mouth guards to help prevent unnecessary dental trauma

- Leon Bassi, dual qualified dental hygienist and dental therapist for investigating the awareness of the International Caries Detection and Assessment System (ICDAS) criteria for the detection of dental caries amongst dental therapists and hygienists

- Nicola Legg, dental nurse for investigating what nurseries have in place regarding tooth friendly snacks and if there were any geographical variations within the city of Portsmouth.

Professor Walls said: 'The Robin Davies Awards luncheon is a wonderful opportunity to celebrate research into oral health care designed and delivered by dental care professionals rather than dentists. This programme is unique globally in providing research support for this group of dental care professionals. It was a great privilege for me as Director of the ODRT to come together with Colgate to celebrate the success of fantastic members of the dental team.

Please visit www.odrt.org.uk/colgate-dcpawards/ to apply for the Robin Davies DCP Awards 2019.

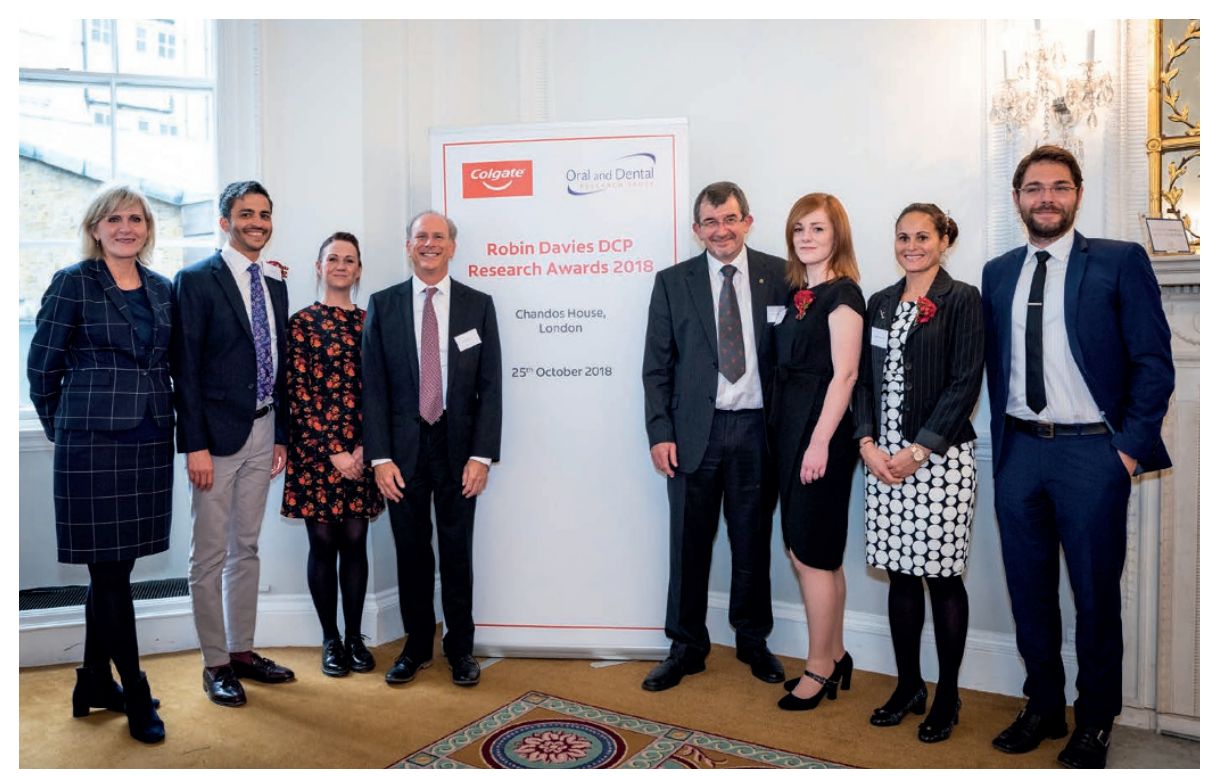

\section{Festive hamper}

After working hard all year, why not treat yourself and colleagues to a connoisseur hamper from Dental Sky?

Containing two bottles of wine and an assortment of festive goodies, the hamper is bound to get everyone into the Christmas spirit.

Valued at more than $£ 70$, it will be dispatched free to customers ordering $£ 750$ worth of goods from Dental Sky between 1-21 December. The offer is limited to one per practice, whilst stocks last.

Furthermore, you'll be able to top up your festive hamper with some treats from Dental Sky's loyalty programme that rewards customers with one loyalty point for every $£ 1$ spent.

Placing a $£ 750$ order would automatically qualify you for a Cadbury's Treasure Box of chocolates, or for an additional $£ 50$, you would have enough for a Green \& Blacks Chocolate and Wine gift set. If you've a large team to treat, then 1,000 points will qualify you for a pack of 20 Toblerone bars.

More information is available by visiting www.dentalsky.com.

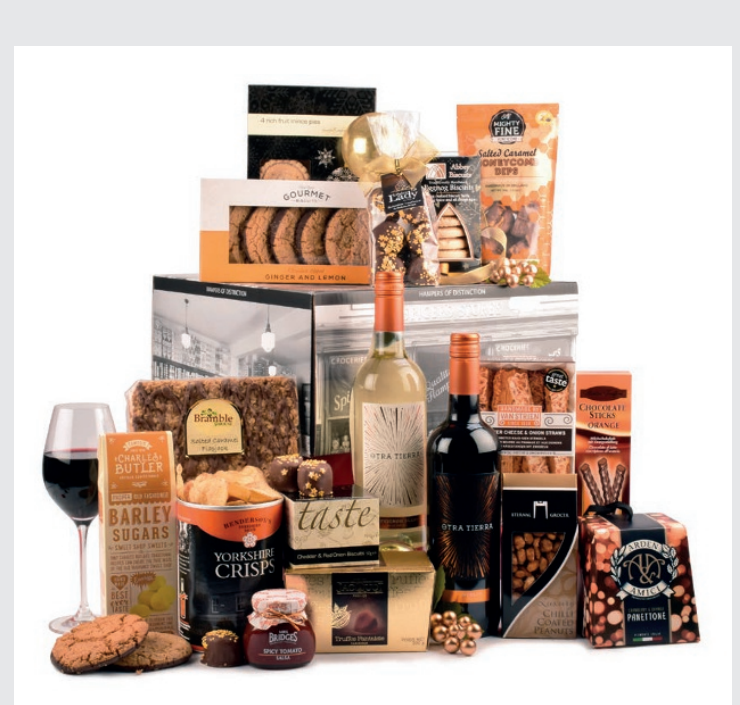

